\title{
EXTENSION OF QUASISYMMETRIC AND LIPSCHITZ EMBEDDINGS OF THE REAL LINE INTO THE PLANE
}

\author{
PEKKA TUKIA
}

1. For the definitions of the terms Lipschitz embedding, Lipschitz homeomorphism and L-embedding, we refer to [7, Section 1].

If $f: X \rightarrow R^{2}, X \subset R^{2}$, is an embedding, we say that $f$ is a quasisymmetric embedding if there is $H \geqq 1$ such that

$$
|f(b)-f(x)| \leqq H|f(a)-f(x)|
$$

provided $a, b, x \in X$ and $|b-x| \leqq|a-x|$; cf. [8], where $f$ was said to be weakly quasisymmetric if this is true. We say also that $f$ is H-quasisymmetric if we wish to emphasize $H$. If $X=R=f(X)$, this definition coincides with the usual definition of a quasisymmetric (and $H$-quasisymmetric) mapping except that $f$ may be also decreasing. Note that an $L$-embedding is always $L^{2}$-quasisymmetric.

2. We prove in this paper the following theorem whose quasiconformal part is more or less known $[4,6]$, although we have not found it in an exactly equivalent form.

Theorem. Let $f: R \rightarrow R^{2}$ be an H-quasisymmetric embedding. Then there is an extension $F$ of $f$ to a K-quasiconformal homeomorphism of $R^{2}$ which is continuously differentiable outside $R$ and where $K$ depends only on $H$. If $f$ is an L-embedding, then $F$ is an $L^{\prime}$-homeomorphism where $L^{\prime}$ depends only on $L$.

We proved a variant of the Lipschitz part of the theorem in [7], where the proof was an explicit geometric construction, based on a certain compactness property of Lipschitz embeddings. The proof we offer here is analytic in character, the main tools being Riemann's mapping theorem and the Beurling-Ahlfors extension of a quasisymmetric mapping. This proof is also much shorter than the proof of [7]. However, it is questionable whether it is fundamentally simpler, so powerful are the theorems on which it is based.

We remark that the construction of [7] would, with minor modifications and with some results of [8], have given also the quasiconformal part of the theorem. Then an easy argument ( $1^{\circ}$ of Section 12 of [7]) gives also the Lipschitz part. How- 
ever, we were not aware of this possibility when writing [7]. If this had been done, we would have had as a corollary a new, geometric proof of the following theorem, proved by Ahlfors [1] using analytic methods. (For the definition of the term "bounded turning", see Section 4.)

Let $S \subset R^{2}$ and assume that $S \cup\{\infty\}$ is a topological circle. Then $S$ is the image $g(R)$ of the real line under a quasiconformal map $g$ of $R^{2}$ if and only if $S$ is of bounded turning.

This would follow since by [8, 4.9], combined with a normal family argument using [8, Section 3], there is a quasisymmetric embedding $f: R \rightarrow R^{2}$ such that $f(R)=S$.

We now proceed to the proof. For further discussion, see Section 7.

3. The Beurling-Ahlfors extension. Let $g: R \rightarrow R$ be an increasing homeomorphism and let $U=\{z \in C: \operatorname{im} z>0\}$. One can extend $g$ to a homeomorphism $B_{g}$ of $\mathrm{cl} U$ by setting $B_{g}=g$ in $R$ and for $(x, y) \in U$

$$
B_{g}(x, y)=\frac{1}{2}(\alpha+\beta)+\frac{1}{2}(\alpha-\beta) i
$$

where

$$
\alpha(x, y)=\int_{0}^{1} g(x+t y) d t, \quad \beta(x, y)=\int_{0}^{1} g(x-t y) d t .
$$

This is the Beurling-Ahlfors extension [2, Section 6] of $g$. It is always a homeomorphism of $\mathrm{cl} U$ and it is continuously differentiable in $U$. In addition, if $g$ is $H$-quasisymmetric, $B_{g}$ is $8 H$-quasiconformal (Reed [3]). We need the following result. Let $\varphi: R^{2} \rightarrow R^{2}$ be affine, $\varphi(z)=a z+b$, where $a, b \in R, a>0$. Then $\varphi(R)=R$ and we have

$$
B_{g \circ \varphi}=B_{g} \circ \varphi, \text { and } B_{\varphi \circ g}=\varphi \circ B_{g},
$$

where we have denoted $\varphi \mid R$ also by $\varphi$. Equations (3) are an immediate consequence of (1) and (2).

If $A: R^{2} \rightarrow R^{2}$ is a linear mapping, we denote

$$
\begin{aligned}
|A| & =\max _{|x|=1}|A(x)| \\
l(A) & =\min _{|x|=1}|A(x)| .
\end{aligned}
$$

A mapping is normalized if it fixes 0,1 and $\infty$. (If a map $g$ is undefined at $\infty$, we set $g(\infty)=\infty$.) The derivative of a map $h$ is $D h$. Now we can state

Lemma 1. Let $g: R \rightarrow R$ be H-quasisymmetric. Then

(a) there is $L_{1}=L_{1}(H) \geqq 1$ such that $B_{g} \mid U$ is an $L_{1}$-homeomorphism of $U$ in the hyperbolic metric of $U$, and 
(b) there is $L_{2}=L_{2}(H) \geqq 1$ such that, if $g$ is in addition normalized,

$$
1 / L_{2} \leqq l\left(D B_{g}(1 / 2,1 / 2)\right) \leqq\left|D B_{g}(1 / 2,1 / 2)\right| \leqq L_{2} .
$$

Proof. For (a) see Ahlfors [1, p, 293]. Let $(x, y) \in U$ and let $\left(x^{\prime}, y^{\prime}\right)=B_{g}(x, y)$. Then (a) implies

$$
1 / L_{1} \leqq y l\left(D B_{g}(x, y)\right) / y^{\prime} \leqq y\left|D B_{g}(x, y)\right| / y^{\prime} \leqq L_{1} .
$$

Now, if $g$ is $H$-quasisymmetric and normalized, $B_{g}$ is $8 H$-quasiconformal and normalized. Then the compactness properties of quasiconformal mappings imply that there is $M=M(H) \geqq 1$ such that $\operatorname{im} B_{g}(1 / 2,1 / 2) \in[1 / M, M]$ whenever $g$ is $H$-quasisymmetric and normalized. It follows by (4) that (b) is true with $L_{2}=2 M L_{1}$.

4. Let $c \geqq 1$. We say that an (open or closed) arc $J \subset R^{2}$ is of $c$-bounded turning if, whenever $a, b \in J$,

$$
\operatorname{diam}\left(J^{\prime}\right) \leqq c|a-b|,
$$

where $J^{\prime} \subset J$ is the subarc with endpoints $a$ and $b$. We let $\mathscr{F}_{c}$ be the family of normalized embeddings $g$ : cl $U \rightarrow R^{2}$ such that $g \mid U$ is conformal and $g(R)$ is of $c$-bounded turning. There is $K=K(c)$ such that every $g \in \mathscr{F}_{c}$ can be extended to a $K$-quasiconformal homeomorphism of $R^{2}$ (cf. [1]). It follows that $\mathscr{F}_{c}$ is compact: if $g_{1}, g_{2}, \ldots \in \mathscr{F}_{c}$, there is a subsequence $g_{n(1)}, g_{n(2)}, \ldots$ such that there is $\lim g_{n(i)} \in \mathscr{F}_{c}$.

Lemma 2. Let $X \subset U$ be compact and let $c \geqq 1$. Then there is $L_{3}=L_{3}(X, c) \geqq 1$ such that the derivative $g^{\prime}(z)$ satisfies

$$
1 / L_{3} \leqq\left|g^{\prime}(z)\right| \leqq L_{3}
$$

for every $g \in \mathscr{F}_{c}$ and $z \in X$.

Proof. We choose a closed path $\gamma:[0,1] \rightarrow U$ such that $\gamma([0,1]) \cap X=\emptyset$ and that for every $z \in X$ the index of $z$ with respect to $\gamma$ is 1 . Then

$$
g^{\prime}(z)=\frac{1}{2 \pi i} \int_{\gamma} \frac{g(\zeta) d \zeta}{(\zeta-z)^{2}}
$$

for every $z \in X$ and $g \in \mathscr{F}_{c}$. Now (6) and the compactness of $\mathscr{F}_{c}$ imply immediately that there is $L_{3}$ for which the right inequality of (5) is valid. If there is no such $L_{3}$ for which the left inequality is also valid, we can find functions $g, g_{1}, g_{2}, \ldots \in \mathscr{F}_{c}$ and points $z, z_{1}, z_{2}, \ldots \in X$ such that $\lim g_{i}=g, \lim z_{i}=z$ and $\lim g_{i}^{\prime}\left(z_{i}\right)=0$. However, $g^{\prime}(z) \neq 0$. This and (6) now imply a contradiction.

5. Let $g: R \rightarrow R^{2}$ be a normalized embedding such that if we set $g(\infty)=\infty$, $g$ is continuous at $\infty$. Let $C_{1}$ and $C_{2}$ be the components of $R^{2} \backslash g(R)$. We choose the notation in such a way that there is an orientation preserving homeomorphism $G: \mathrm{cl} U \rightarrow \mathrm{cl} C_{1}$ extending $g$. Now we construct a canonical extension 
$F_{g}$ of $g$ to a homeomorphism of $R^{2}$. We give the definition of $F_{g}$ only in the upper half-plane $U$; the case of the lower half-plane is analogous.

There is a well-defined homeomorphism $A_{g}: \mathrm{cl} U \rightarrow \mathrm{cl} C_{1}$ which is normalized and conformal in $U$. Consider the map $\bar{g}=A_{g}^{-1} \circ g$ which is an increasing homeomorphism of $R$; thus the Beurling-Ahlfors extension $B_{\bar{g}}$ is a homeomorphism of cl $U$ extending $\bar{g}$. We set now

$$
F_{g} \mid \mathrm{cl} U=A_{g} \circ B_{\bar{g}},
$$

$F_{g}$ being defined similarly in $R^{2} \backslash U$. We have $F_{g} \mid R=A_{g} \circ \bar{g}=A_{g} \circ A_{g}^{-1} \circ g=g$; thus $F_{g}$ is indeed an extension of $g$.

Let then $\varphi: R \rightarrow R$ be an increasing affine map and $\psi: R^{2} \rightarrow R^{2}$ be a conformal affine map, and assume that $\psi g \varphi$ is normalized. We show

$$
F_{\psi g \varphi}=\psi \circ F_{g} \circ \varphi
$$

(where on the right side $\varphi$ is extended to the unique conformal affine map extending $\varphi$ ). This is the fundamental property of $F_{g}$ which makes the proof of our theorem possible. We obscrve first that, if $h=\psi g \varphi$,

$$
A_{h}=\psi \circ A_{g} \circ \bar{\varphi}
$$

where $\bar{\varphi}=A_{g}^{-1} \circ \psi^{-1} \circ A_{h}$ is a conformal affine map of cl $U$. Let $\bar{h}=A_{h}^{-1} \circ h$. Then

$$
\bar{h}=\bar{\varphi}^{-1} \circ A_{g}^{-1} \circ \psi^{-1} \circ \psi \circ g \circ \varphi=\bar{\varphi}^{-1} \circ A_{g}^{-1} \circ g \circ \varphi=\bar{\varphi}^{-1} \circ \bar{g} \circ \varphi .
$$

Consequently, by (3), $B_{\bar{h}}=\bar{\varphi}^{-1} \circ B_{\bar{g}} \circ \varphi \mid \mathrm{cl} U$. Thus, by (9),

$$
F_{h}\left|\operatorname{cl} U=A_{h} \circ B_{\bar{h}}=\psi \circ A_{g} \circ \bar{\varphi} \circ \bar{\varphi}^{-1} \circ B_{\bar{g}} \circ \varphi\right| \operatorname{cl} U=\psi \circ F_{g} \circ \varphi \mid \operatorname{cl} U .
$$

In the same manner one sees that (8) is valid also in $R^{2} \backslash U$.

If now $g$ is a quasisymmetric embedding of $R$ into $R^{2}$, necessarily $\lim _{|t| \rightarrow \infty}|g(t)|=\infty \quad([8,2.1$ and 2.16]) and thus the above discussion is valid for all normalized quasisymmetric maps. Note that, if $g$ is $H$-quasisymmetric, then $g(R)$ is of $2 H$-bounded turning.

Lemma 3. Let $H \geqq 1$. There is a number $L_{4}=L_{4}(H) \geqq 1$ such that if $f: R \rightarrow R^{2}$ is a normalized $H$-quasisymmetric embedding, then the extension $F_{f}$ is continuously differentiable in $R^{2} \backslash R$ and satisfies

$$
\frac{|f(x+y)-f(x-y)|}{\left|2 y L_{4}\right|} \leqq l\left(D F_{f}(x, y)\right) \leqq\left|D F_{f}(x, y)\right| \leqq \frac{|f(x+y)-f(x-y)|}{|2 y|} L_{4}
$$

for $(x, y) \in R^{2} \backslash R$.

Proof. Choose $(x, y) \in R^{2} \backslash R$. We can assume that $y>0$. Let $\varphi$ be the increasing affine map of $R$ such that $\varphi(0)=x-y$ and $\varphi(1)=x+y$; we denote by $\varphi$ also the extension of $\varphi$ to a conformal affine map of $R^{2}$. Let $\psi$ be the 
conformal affine map of $R^{2}$ such that $\psi(f(x-y))=0$ and $\psi(f(x+y))=1$. Then $g=\psi f \varphi$ is normalized and by $(8)$

$$
D F_{f}(x, y)=D \psi^{-1} \circ D F_{g}(1 / 2,1 / 2) \circ D \varphi^{-1} .
$$

Now $D \varphi$ and $D \psi$ (which are constants) are similarities, and $D \varphi$ multiplies distances by the factor $2 y$ and $D \psi$ multiplies them by the factor $1 /|f(x+y)-f(x-y)|$. Thus the lemma is true if we can show that there is $L_{4}=L_{4}(H)$ such that

$$
1 / L_{4} \leqq l\left(D F_{g}(1 / 2,1 / 2)\right) \leqq\left|D F_{g}(1 / 2,1 / 2)\right| \leqq L_{4} .
$$

Observe that $g$ is normalized and $H$-quasisymmetric. We show that, in fact, there is $L_{4}=L_{4}(H)$ such that (10) is true for all normalized and $H$-quasisymmetric $g$. By (7),

$$
D F_{g}(1 / 2,1 / 2)=D A_{g}\left(B_{\bar{g}}(1 / 2,1 / 2)\right) \circ D B_{\bar{g}}(1 / 2,1 / 2) .
$$

Here $\bar{g}=A_{g}^{-1} \circ g$ is normalized. It is also $H^{\prime}$-quasisymmetric for some $H^{\prime}=H^{\prime}(H)$. To see this, observe first that $A_{g} \in \mathscr{F}_{2 H}$ since $g(R)$ is of $2 H$-bounded turning. Thus $A_{g}$, and hence also $A_{g}^{-1}$, can be extended to a $K(H)$-quasiconformal homeomorphism of $R^{2}$. But a $K(H)$-quasiconformal homeomorphism of $R^{2}$ is $H_{1}$-quasisymmetric for some $H_{1}=H_{1}(K(H))=H_{1}(H)$. This follows by a normal family argument or by $[9,2.4]$. But then $A_{g}^{-1} \circ g$ is $H^{\prime}$-quasisymmetric for some $H^{\prime}=H^{\prime}\left(H_{1}, H\right)=H^{\prime}(H)$ by $[8,2.16$ and 2.2].

Let $X=\left\{B_{h}(1 / 2,1 / 2): h: R \rightarrow R\right.$ is normalized and $H^{\prime}$-quasisymmetric $\}$. Then a normal family argument shows that $X=X(H) \subset U$ is compact (observe that $B_{g}$ depends continuously on $g$ ). Now (11) and Lemmas 1 and 2 imply that there are $L_{2}=L_{2}\left(H^{\prime}\right)=L_{2}(H)$ and $L_{3}=L_{3}(X, 2 H)=L_{3}(H)$ such that (11) is true with $L_{4}=L_{2} L_{3}=L_{4}(H)$.

6. The proof of the main theorem is now easy. We can assume that $f$ is normalized, possibly by increasing $L^{\prime}$ for non-normalized $f$. Obviously, $F_{f}$ is continuously differentiable outside $R$. Thus $F_{f} \mid R^{2} \backslash R$ is $L_{4}^{2}$-quasiconformal, which implies that also $F_{f}$ is $L_{4}^{2}$-quasiconformal since $R$ is a removable singularity for quasiconformal maps of $R^{2}$. Assume then that $f$ is an $L$-embedding. We observe that $f$ is $L^{2}$-quasisymmetric and thus we can apply Lemma 3, which implies that $1 / L L_{4} \leqq 1\left(D F_{f}(x, y)\right) \leqq\left|D F_{f}(x, y)\right| \leqq L L_{4} \quad$ if $\quad(x, y) \in R^{2} \backslash R$. It follows that $F_{f}$ is an $L^{\prime}$-embedding where $L^{\prime}=L L_{4}=L^{\prime}(L)$.

7. Actually, Theorem A of [7] and the present theorem consider a slightly different situation since in [7] we considered a Lipschitz embedding $f: S=\partial I^{2} \rightarrow R^{2}$. However, these two cases can be fairly easily reduced to each other by means of the following observation, due to J. Väisälä. For every $L \geqq 1$ there is $K=K(L) \geqq 1$ such that if $f: R \rightarrow R^{2}$ or $f: R^{2} \rightarrow R^{2}$ is an L-embedding in the euclidean metric with $f(0)=0$, then $f$ is a K-embedding in the spherical metric, and vice versa. This 
follows easily by the expression $|d z| /\left(1+|z|^{2}\right)$ for the spherical metric. This observation would have simplified the discussion in Section 12 of [7], where we extended $f: S \rightarrow R^{2}$ outside $S$. We refer to this discussion for the details of how to reduce the theorems to each other (apart from the requirement that the extensions are PL or continuously differentiable outside $S$ or $R$ ).

Presumably one would get by this method also the extension for Lipschitz or quasisymmetric embeddings of arcs into $R^{2}$ (cf. Theorem B of [7]) since quasiconformal arcs can be characterized in a manner similar to quasiconformal circles (Rickman [5]).

Finally, I am indebted to J. Luukkainen and J. Väisälä for reading the manuscript and for valuable remarks.

Added in proof. After this paper was completed, I received the paper "Hardy spaces, $A_{\infty}$, and singular integrals on chord-arc domains" by D.S,Jerison and C,E.Kenig whose Proposition 1.13. is equivalent to the Lipschitz part of the theorem of this paper.

\title{
References
}

[1] Ahlfors, L. V.: Quasiconformal reflections. - Acta Math. 109, 1963, 291-301.

[2] Beurling, A., and L. Ahlfors: The boundary correspondence under quasiconformal mappings. - Acta Math. 96, 1956, 125-142.

[3] REED, T. J.: Quasiconformal mappings with given boundary values. - Duke Math. J. 33, 1966, $459-464$.

[4] REED, T. J.: On the boundary correspondence of quasiconformal mappings of domains bounded by quasi circles. - Pacific J. Math. 28, 1969, 653-661.

[5] Rickman, S.: Characterization of quasiconformal arcs. - Ann. Acad. Sci. Fenn. Ser. A I Math. 395, 1966, 1-30.

[6] Rickman, S.: Quasiconformally equivalent curves. - Duke Math. J. 36, 1969, 387-400.

[7] Tukia, P.: The planar Schönflies theorem for Lipschitz maps. - Ann. Acad. Sci. Fenn. Ser. A I Math. 5, 1980, 49-72.

[8] Tukia, P., and J. VÄIs̈̈LÄ: Quasisymmetric embeddings of metric spaces. - Ibid. 5, 1980, 97 114.

[9] VÄISÄLÄ, J.: Quasisymmetric embeddings in euclidean spaces. - Trans. Amer. Math. Soc. 264, 1981, 191-204.

\author{
University of Helsinki \\ Department of Mathematics \\ SF-00100 Helsinki 10 \\ Finland
}

Received 9 May 1980 